\title{
Technical note: Validation of a commercial system for the continuous and automated monitoring of dairy cow activity
}

\author{
E. Tullo, ${ }^{*}$ I. Fontana, ${ }^{* 1}$ D. Gottardo, ${ }^{*}$ K. H. Sloth, $\dagger$ and M. Guarino* \\ *Department of Veterinary and Technological Sciences for Food Safety, Faculty of Veterinary Medicine, Università degli Studi, Via Celoria 10, \\ 20133 Milan, Italy \\ †GEA Farm Technologies GmbH, Siemensstraße 2559199 Bönen, Germany
}

\section{ABSTRACT}

Current farm sizes do not allow the precise identification and tracking of individual cows and their health and behavioral records. Currently, the application of information technology within intensive dairy farming takes a key role in proper routine management to improve animal welfare and to enhance the comfort of dairy cows. An existing application based on information technology is represented by the GEA CowView system (GEA Farm Technologies, Bönen, Germany). This system is able to detect and monitor animal behavioral activities based on positioning, through the creation of a virtual map of the barn that outlines all the areas where cows have access. The aim of this study was to validate the accuracy, sensitivity, and specificity of data provided by the CowView system. The validation was performed by comparing data automatically obtained from the CowView system with those obtained by a manual labeling procedure performed on video recordings. Data used for the comparisons were represented by the zone-related activities performed by the selected dairy cows and were classified into 2 categories: activity and localization. The duration in seconds of each of the activities/localizations detected both with the manual labeling and with the automated system were used to evaluate the correlation coefficients among data; and subsequently the accuracy, sensitivity, specificity, and positive and negative predictive values of the automated monitoring system were calculated. The results of this validation study showed that the CowView automated monitoring system is able to identify the cow localization/position (alley, trough, cubicles) with high reliability in relation to the zone-related activities performed by dairy cows (accuracy higher than 95\%). The results obtained support the CowView system as

Received February 10, 2016.

Accepted May 20, 2016.

${ }^{1}$ Corresponding author: ilaria.fontana@unimi.it an innovative potential solution for the easier management of dairy cows.

Key words: dairy cow, validation, sensor, automated monitoring system

\section{Technical Note}

The current increase in the global demand for dairy products means that the dairy industry has undergone profound changes over recent decades (Barkema et al., 2015). Economic pressures, technological innovations, demographic shifts, consumer expectations, and an evolving regulatory framework have contributed to the impetus for changes in the global dairy industry (Barkema et al., 2015), aimed at the maximization of productivity and efficiency (Gerber et al., 2011). Moreover, the consolidation of farms has resulted in larger herd sizes (von Keyserlingk et al., 2013), with relevant effects on the health and welfare of dairy cows and on management practices and systems for dairy herds (Barkema et al., 2015). However, current farm sizes do not allow the precise identification and tracking of individual cows and their health and behavioral records.

Monitoring the behavior of dairy cows is useful to assess their welfare, health status, and comfort at farm level (Mattachini et al., 2013). Indeed, changes in behavior are clear indicators of dairy cows' health and welfare problems and therefore they can be used as input to an early warning system. The time spent by the cows lying or feeding plays an important role in terms of milk production (Fregonesi et al., 2007; Mattachini et al., 2011); therefore, knowing their position is important to monitor their behavioral patterns and activity (Huhtala et al., 2007) to obtain information to control and monitor cows' health status and productivity.

However, continuous monitoring requires a lot of manpower/labor and is time consuming (Fontana et al., 2015a). For this reason, precision livestock farming approaches can combine information technology (IT) into online automated tools that can be used to control, monitor, and model the behavior of animals and their biological responses (Tullo et al., 2013). 
Since the 1980s, progress has been made on devices that measure health indicators in, up, on, or from an individual cow (Hogeveen et al., 2010). Examples of sensors used on dairy cows include milk electrical conductivity, milk color sensors, acceleration sensors attached to the cow's leg, and $\mathrm{pH}$ sensors. A sensor system consists of the device itself plus the software that processes the data to produce information or advice (Rutten et al., 2013). Several studies have confirmed the feasibility of the use of information technology, achieving excellent results in the identification and localizations of the animals, feeding pattern recognition, and estrus detection (Porto et al., 2014).

Nowadays, the application of IT within intensive dairy farming takes a key role in proper routine management to improve animal welfare and to enhance the comfort of dairy cows (Fontana et al., 2015b).

An existing application based on IT is the GEA CowView system (GEA Farm Technologies, Bönen, Germany). The CowView system is an automatic indoor localization system for dairy cattle, providing data on positions and zone-related behavior or activity of tagged animals based on triangulation of very short radio-signals (Ultra-Wide Band) with a precision of 50 cm (CowView system, GEA Farm Technologies).

This system continually records the position of each cow through a connection among sensors placed in the barn and the CowView tag mounted on the cow's collar. This system is able to detect and monitor animal behaviors based on positioning through the creation of a virtual map of the barn that outlines all areas where the cows have access. The output is a data set with the position of the animal characterized by spatial coordinates that allow the identification of behaviors. Indeed, a distinctive feature of this technology is the division of the whole area into sub-areas, which define specific cow behaviors (for example, being at the feeding table corresponds to feeding behavior).

The aim of this study was to validate the accuracy, sensitivity, and specificity of the CowView automatic monitoring system that tracks cow position and behavior.

Data collection consisted of 2 steps for the creation of 2 different data sets to be compared during the validation.

Five selected cows were equipped with the CowView electronic tag and were marked with a yellow letter on both flanks and on the back.

The first step of data collection was automatically performed with the CowView system; it resulted in an output file containing the position of each animal characterized by spatial coordinates. The second step of data collection consisted of video recordings obtained with a camera [Axis P5534 PTZ Dome Network, (Uptime-IT, Svendborg, Denmark), 30 frames per second (fps), and 1,280 $\times 720$ pixels] placed in a top-down perspective under the roof of the experimental barn. Total video recordings lasted for around $42 \mathrm{~h}(150,405$ $\mathrm{s}$, at $30 \mathrm{fps}$ that ended with $4.5 \times 10^{6}$ frames) over 6 d. Successively, recordings were visually checked and manually labeled, by an operator, playing the videos or observing them frame by frame (30 fps; Ismayilova et al., 2013).

These video recordings were visually processed (video labeling), following the marked cows in their behaviors and positions. Checking the video frame by frame allowed the operator to precisely detect the start time of a cow behavior/position. The shooting angles of the camera did not allow the operator to continuously follow the marked cows; therefore, parts of the videos without any marked cow were discarded. When a specific behavior/position was observed in the video, information such as classification, zone, start time, and duration were reported in a data set used as reference for the validation.

Each behavior/position was classified second by second; more precisely, an activity lasting $10 \mathrm{~s}$ was recorded in the data set as 10 repeated activities of $1 \mathrm{~s}$.

The outcome of the video labeling was used as the gold standard for the validation of the accuracy of the system. The validation was performed by comparing both data sets obtained to check the system reliability in localizing the position of the cows and their behavior.

Data used for the validation were represented by the zone-related activities performed by the selected cows and were classified into 2 categories: behavior and position. The "feeding" behavior was considered to be taking place when the cow's head was in the fodder line, both in the manual labeling and in the development of the software. "In bed" behavior was considered to take place during the manual labeling when the cow was in the cubicle, but the system was not able to recognize whether the cows were lying down or not. The validation also included the categories "at the drinker" both as a behavior and a position. The activity was not classified as "drinking" because the CowView system could not identify this behavior.

The CowView system provided information at cow level, classifying each behavior/position second by second, listing in the output the zone, start time, and duration.

The 2 data sets obtained were merged. Only time intervals where data were available from both data sets were used in the evaluation, resulting in a final data 
set of about $37 \mathrm{~h}(132,053 \mathrm{~s}$, at $30 \mathrm{fps}$ that ended up in $3.9 \times 10^{6}$ frames) of video data. The duration in seconds of each behavior/position detected both with the manual labeling and with the automated system was used to evaluate the correlation coefficients among data (PROC CORR, SAS 9.3, SAS Institute Inc., Cary, NC). Subsequently, the data were analyzed by $2 \times 2$ contingency tables (true positives, false negatives, false positives, and true negatives), to estimate the accuracy, sensitivity, specificity, and positive $(\mathbf{P V}+)$ and negative predictive value $(\mathbf{P V}-)$ of the automated monitoring system (Gonen, 2006; Trénel et al., 2009; Vandermeulen et al., 2015).

The parameters evaluated for each activity/localization were as follows:

- Sensitivity, parameter that tests the true positive rate:

$$
\text { Sensitivity }=\frac{\text { true positives }}{\text { true positive }+ \text { false negative }} \text {. }
$$

- Specificity, parameter that tests the true negative rate:

Specificity $=\frac{\text { true negatives }}{\text { true negative }+ \text { false positives }}$.
- Predictive value for a positive result $(\mathrm{PV}+)$, parameter that tests the probability that the CowView detects behavior that cow is actually performing:

$$
\mathrm{PV}+=\frac{\text { true positive }}{\text { true positive }+ \text { false positive }}
$$

- Predictive value for a negative result (PV-): parameter testing the probability that the CowView does not detect behavior that cow is actually not performing:

$$
\mathrm{PV}-=\frac{\text { true negatives }}{\text { true negatives }+ \text { false negatives }} .
$$

- Accuracy: parameter that expresses the proportion of correctly classified behaviors among all events detected:

$$
\text { Accuracy }=\frac{\text { true positive }+ \text { true negative }}{\text { all events }} .
$$

Figures 1 and 2 display graphical examples of comparison between data manually labeled and automatically detected by the CowView system.

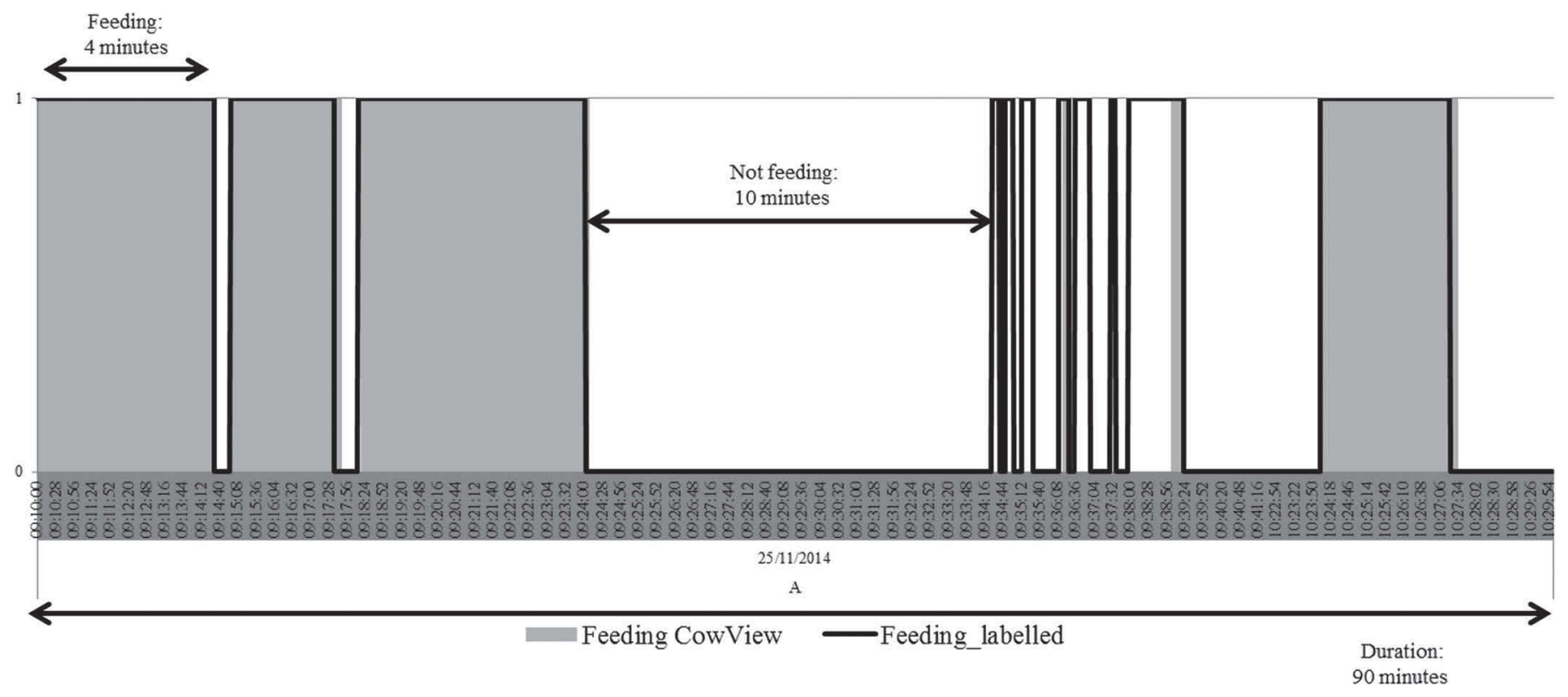

Figure 1. Example of comparison between data manually labeled and automatically detected by the CowView system (GEA Farm Technologies, Bönen, Germany) in relation to the activity of "feeding" on a selected cow and on a selected day. Values of 1 indicate activity detection (manual/automated). 

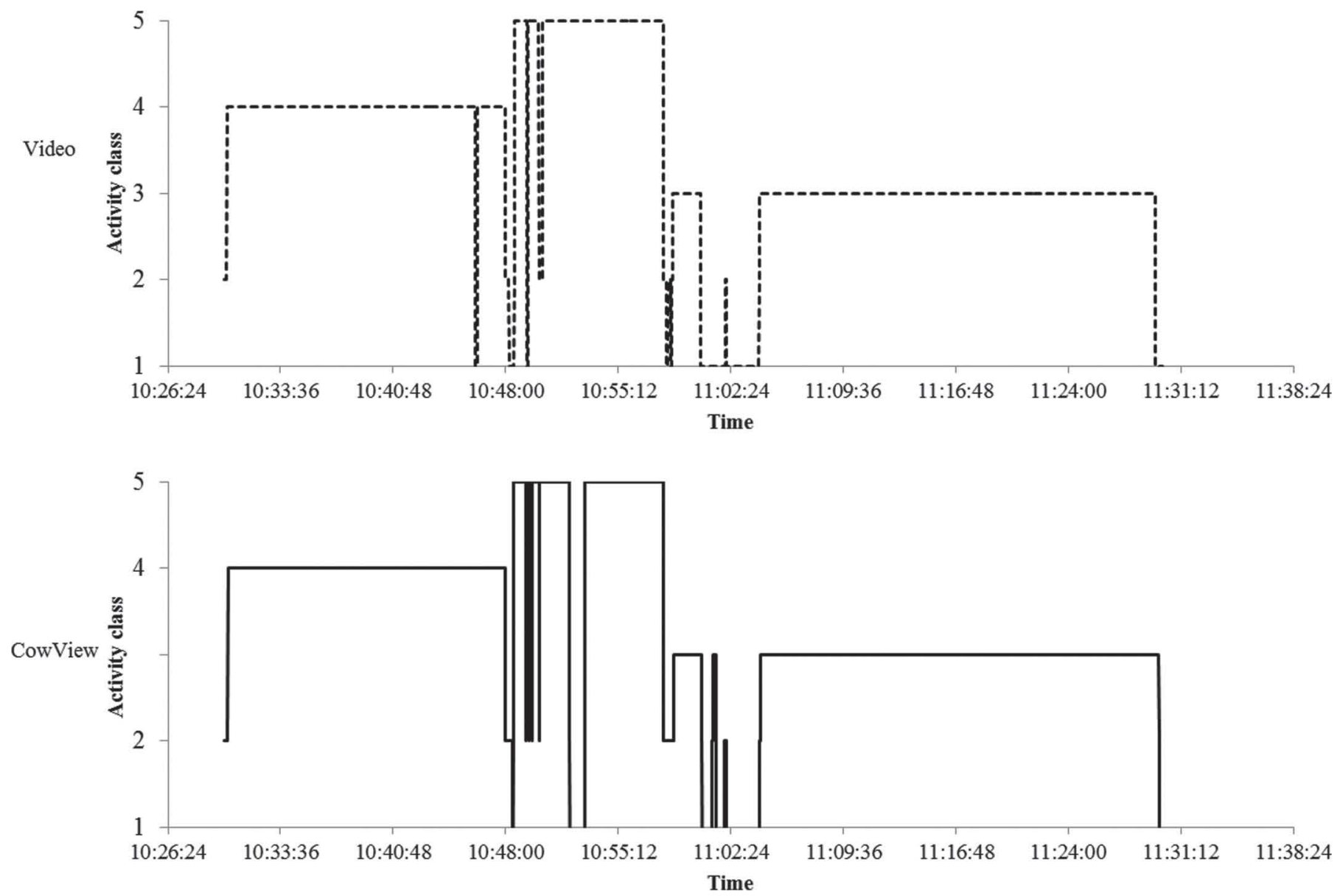

Figure 2. Example of comparison between data manually labeled and automatically detected by the CowView system (GEA Farm Technologies, Bönen, Germany) in relation to the 5 activities and positions during part of the observation period. Activity classes: $1=$ standing in alley, $2=$ walking in alley, $3=$ in cubicle, $4=$ at feeding table, $5=$ at drinker.

Figure 1 shows the relation for feeding behavior between data obtained both with the Cow View system and video labeling. The time interval displayed is about 90 min, chosen randomly across the video validation. Grey parts represent the amount of time (s) spent by the selected cow in the activity of "feeding" detected by the CowView system. Black lines represent the actual time (detected with the manual labeling) spent by that cow doing the specific activity. Grey parts with the black frame represent the results obtained with the automated system that can be overlapped to the reference values (manual labeling). Black lines without gray filling represent a mismatch between automated detection and reference values.

Figure 2 displays the relation between data obtained both with the Cow View system and the video labeling for the 5 activities/positions during part of the observation period. Dotted lines represent data obtained with the video labeling, and black lines identify output data from the CowView system. The figure shows how the 2 outputs fit each other.

The correlation coefficients (Table 1) computed from data obtained from the manual processing of video recordings and from the CowView system were quite high with values above 0.82 , except for the "walking" activity (0.51). The low correlation coefficient obtained for the "walking" indicates the difficulty of the system in detecting this activity without confounding it with other activities estimated on the localization of cows. The other correlation coefficients show how the output of the system fits with the reference values obtained with the manual labeling, indicating the reliability of the automated system. Indeed, the more accurate the cow localization is, the more precise the detection of zone-related activities.

Table 1 also shows the sensitivity, specificity, PV+, PV-, and accuracy of the activity/location detected by the CowView system. 
Table 1. Correlation with reference values, accuracy, sensitivity, specificity, and the positive (PV+) and negative (PV-) predictive value of the output of the CowView system of each activity and position detected

\begin{tabular}{|c|c|c|c|c|c|c|}
\hline Item & $\begin{array}{l}\text { Correlation with } \\
\text { reference values }\end{array}$ & $\begin{array}{l}\text { Sensitivity } \\
\text { (SE) }\end{array}$ & $\begin{array}{l}\text { Specificity } \\
\quad(\mathrm{SE})\end{array}$ & $\begin{array}{l}\mathrm{PV}+ \\
(\mathrm{SE})\end{array}$ & $\begin{array}{l}\mathrm{PV}- \\
(\mathrm{SE})\end{array}$ & $\begin{array}{l}\text { Accuracy } \\
\quad(\mathrm{SE})\end{array}$ \\
\hline \multicolumn{7}{|l|}{ Cow activity } \\
\hline Walking & 0.51 & $\begin{array}{l}0.78 \\
(0.0053)\end{array}$ & $\begin{array}{l}0.97 \\
(0.0005)\end{array}$ & $\begin{array}{l}0.53 \\
(0.0052)\end{array}$ & $\begin{array}{l}0.99 \\
(0.0003)\end{array}$ & $\begin{array}{l}0.96 \\
(0.0006)\end{array}$ \\
\hline \multicolumn{7}{|l|}{ Cow position } \\
\hline Cubicles & 1.00 & $\begin{array}{l}0.95 \\
(0.001)\end{array}$ & $\begin{array}{l}0.99 \\
(0.0004)\end{array}$ & $\begin{array}{l}0.98 \\
(0.0007)\end{array}$ & $\begin{array}{l}0.97 \\
(0.0005)\end{array}$ & $\begin{array}{l}0.97 \\
(0.0004)\end{array}$ \\
\hline Trough & 0.98 & $\begin{array}{l}0.97 \\
(0.0008)\end{array}$ & $\begin{array}{l}0.97 \\
(0.0006)\end{array}$ & $\begin{array}{l}0.94 \\
(0.001)\end{array}$ & $\begin{array}{l}0.98 \\
(0.0005)\end{array}$ & $\begin{array}{l}0.97 \\
(0.0005)\end{array}$ \\
\hline At the drinker & 0.88 & $\begin{array}{l}0.85 \\
(0.0039)\end{array}$ & $\begin{array}{l}0.98 \\
(0.0004)\end{array}$ & $\begin{array}{l}0.70 \\
(0.0045)\end{array}$ & $\begin{array}{l}0.99 \\
(0.0003)\end{array}$ & $\begin{array}{l}0.97 \\
(0.0005)\end{array}$ \\
\hline
\end{tabular}

${ }^{1}$ Level of significance of correlation: $P<0.001$.

The high values in sensitivity and specificity (higher than $95 \%$ ) for activity/localization indicate the high rate of true positives and true negatives detected by the CowView system. In other words, the CowView can correctly detect, compared with reference values, whether the position of the cow and its behavior is occurring or not. This is especially true for the "cubicles" and "trough" location, and for the related "in bed" and "feeding" activities.

The high values of $\mathrm{PV}+$ (predictive value for a positive result) indicate the increased probability that the CowView detects a behavior that the cow is actually performing. On the other hand, high values of predictive values for a negative result $(\mathrm{PV}-\geq 93 \%)$ mean that the system is able to "not detect" behavior that cow is actually not performing, thus increasing the reliability of the system itself.

The reliability of the system is also confirmed by the high values of accuracy that reached values of $97 \%$ for the majority of the positional output.

The results of this validation study showed that the CowView automatic monitoring system is able to identify the cow position (alley, trough, cubicles) with high reliability in relation to the behavior/activity performed by dairy cows. Parameters that could be improved in the software optimization phase are "walking" and "standing," since these activities were determined with the lowest correlation. The preliminary results obtained are overall very encouraging, even if the accuracy does not reach $100 \%$. The software provides an innovative solution and it might be a valuable tool for the management of large herds of dairy cattle. Further design optimization of the CowView installation and relative validation will be necessary if the accuracy and the reliability of the system are to be improved.

\section{ACKNOWLEDGMENTS}

This project was funded by the European project no: 311825 EU-PLF (Animal and farm-centric approach to precision livestock farming in Europe) and co-financed by the European Commission (Brussels, Belgium).

\section{REFERENCES}

Barkema, H. W., M. A. G. von Keyserlingk, J. P. Kastelic, T. J. G. M. Lam, C. Luby, J. P. Roy, S. J. LeBlanc, G. P. Keefe, and D. F. Kelton. 2015. Invited review: Changes in the dairy industry affecting dairy cattle health and welfare. J. Dairy Sci. 98:7426-7445.

Fontana, I., E. Tullo, A. Butterworth, and M. Guarino. 2015a. An innovative approach to predict the growth in intensive poultry farming. Comput. Electron. Agric. 119:178-183.

Fontana, I., E. Tullo, D. Gottardo, C. Bahr, S. Viazzi, K. H. Sloth, and M. Guarino. 2015b. Validation of a commercial system for the continuous and automated monitoring of dairy cows activity. Pages 93-102 in Proc. Precision Livestock Farming 2015-Papers Presented at the 7th European Conference on Precision Livestock Farming, ECPLF 2015.

Fregonesi, J. A., C. B. Tucker, and D. M. Weary. 2007. Overstocking reduces lying time in dairy cows. J. Dairy Sci. 90:3349-3354.

Gerber, P., T. Vellinga, C. Opio, and H. Steinfeld. 2011. Productivity gains and greenhouse gas emissions intensity in dairy systems. Livest. Sci. 139:100-108.

Gonen, M. 2006. Receiver operating characteristic (ROC) curves. SAS Users Group International 31:210-231.

Hogeveen, H., C. Kamphuis, W. Steeneveld, and H. Mollenhorst. 2010 Sensors and clinical mastitis - The quest for the perfect alert. Sensors (Basel) 10:7991-8009.

Huhtala, A., K. Suhonen, P. Mäkelä, M. Hakojärvi, and J. Ahokas. 2007. Evaluation of instrumentation for cow positioning and tracking indoors. Biosys. Eng. 96:399-405.

Ismayilova, G., A. Costa, I. Fontana, D. Berckmans, and M. Guarino. 2013. Labelling the behaviour of piglets and activity monitoring from video as a tool of assessing interest in different environmental enrichments. Ann. Anim. Sci. 13:611-621.

Mattachini, G., A. Antler, E. Riva, A. Arbel, and G. Provolo. 2013. Automated measurement of lying behavior for monitoring the comfort and welfare of lactating dairy cows. Livest. Sci. 158:145-150. 
Mattachini, G., E. Riva, and G. Provolo. 2011. The lying and standing activity indices of dairy cows in free-stall housing. Appl. Anim. Behav. Sci. 129:18-27.

Porto, S. M. C., C. Arcidiacono, A. Giummarra, U. Anguzza, and G. Cascone. 2014. Localisation and identification performances of a real-time location system based on ultra wide band technology for monitoring and tracking dairy cow behaviour in a semi-open freestall barn. Comput. Electron. Agric. 108:221-229.

Rutten, C. J., A. G. J. Velthuis, W. Steeneveld, and H. Hogeveen. 2013. Invited review: Sensors to support health management on dairy farms. J. Dairy Sci. 96:1928-1952.

Trénel, P., M. B. Jensen, E. L. Decker, and F. Skjøth. 2009. Technical note: Quantifying and characterizing behavior in dairy calves using the IceTag automatic recording device. J. Dairy Sci. 92:3397-3401.
Tullo, E., I. Fontana, and M. Guarino. 2013. Precision livestock farming: An overview of image and sound labelling. Pages 30-38 in Proc. Precision Livestock Farming 2013-Papers Presented at the 6th European Conference on Precision Livestock Farming, ECPLF 2013.

Vandermeulen, J., C. Bahr, E. Tullo, I. Fontana, S. Ott, M. Kashiha, M. Guarino, C. P. Moons, F. A. Tuyttens, and T. Niewold. 2015. Discerning pig screams in production environments. PLoS ONE 10:e123111.

von Keyserlingk, M., N. Martin, E. Kebreab, K. Knowlton, R. Grant, M. Stephenson, C. Sniffen, J. Harner, A. Wright, and S. Smith 2013. Invited review: Sustainability of the US dairy industry. J. Dairy Sci. 96:5405-5425. 